\title{
DISKUSSION
}

\section{Direktwahl als direkte Demokratie? Überlegungen anlässlich Frank Deckers Forderung nach „Direktwahl der Ministerpräsidenten“" in Heft 2/2013 der ZParl}

\author{
Wolfgang Zeh
}

Das parlamentarische Regierungssystem nach dem Grundgesetz, also die personelle Besetzung der wichtigsten Staatsämter durch gewählte Mehrheiten im Parlament, hat sich in Deutschland durchgesetzt, aber nicht mit Leichtigkeit. Noch heute, nach bald 65 Jahren einer für hiesige Verhältnisse historisch einzigartigen Erfolgsgeschichte, taucht in der politischen und staatstheoretischen Diskussion immer wieder einmal der Gedanke auf, wie viel demokratischer es doch wäre, könnten die Bürger durch allgemeine Wahlen direkt über die Personen entscheiden, von welchen sie regiert oder repräsentiert werden wollen. Ein beliebtes Objekt solcher Erwägungen ist der Bundespräsident ${ }^{1}$, gelegentlich animiert durch einen Amtsinhaber selbst wie Horst Köhler.

(1) Bei Decker sollen es nun - im Gefolge von Arnims - die Regierungschefs in den Bundesländern sein. An prominenter Stelle beruft er sich dafür auf Wilhelm Hennis und Theodor Eschenburg, deren Aufsätze aus den fünfziger Jahren heute bedauerlicherweise keine Rolle mehr spielten. ${ }^{2}$ In diesem Zusammenhang darf auf das aktuelle Referendum in der Schweiz (9. Juni 2013) aufmerksam gemacht werden, mit dem ein Antrag der Konservativen auf Direktwahl des Regierungschefs abgelehnt wurde.

Als Grundton schwingt in solchen Überlegungen regelmäßig die Vorstellung mit, Demokratie, wörtlich Herrschaft des Volkes, sei „eigentlich“ erst dann ganz bei sich selbst, wenn „das Volk“ möglichst viel im Staat selbst entscheide und möglichst wenig an Repräsentanten übertrage. Diese letzteren, mit ihnen die Parlamente und die dort vertretenen politischen Parteien, erscheinen dann als Ersatz und Umweg für etwas, das besser, direkter, volks- und bürgernäher sein müsste. Und wenn der Erkenntnis nicht mehr auszuweichen ist, dass das „Ideal“ der Identität von Regierenden und Regierten - von Ernst Fraenkel einst als „vulgärdemokratisch"3 gebrandmarkt - entweder nicht effizient oder aber nicht gewaltfrei verwirklicht werden kann, lässt sich immer noch die Forderung erheben, bestimmte Elemente innerhalb des Repräsentativsystems müssten stärker "direkt“ organisiert sein, indem die repräsentative Legitimationskette vom Bürger zum Staatshandeln hin wenigstens verkürzt

1 Eine gründliche und kritische Aufarbeitung dieser Diskussion bietet Peter Rütters, Direktwahl des Bundespräsidenten: Sehnsucht nach präsidentieller Obrigkeit?, in: ZParl, 44. Jg. (2013), H. 2, S. $276-295$.

2 Vgl. Frank Decker, Direktwahl der Ministerpräsidenten: Begründung, Ausgestaltung und Umsetzbarkeit eines Wechsels der Regierungsform in den Ländern, in: ZParl, 44. Jg. (2013), H. 2, S. 296 - 314, S. 296.

3 Ernst Fraenkel, Historische Vorbelastungen des deutschen Parlamentarismus, in: ders., Deutschland und die westlichen Demokratien, erweiterte Ausgabe, Frankfurt am Main 1991, S. 23 - 47, S. 32. 
werde. Mit einer Direktwahl der Ministerpräsidenten wäre ein Glied jener Kette - ihre Wahl durch Mitglieder des jeweiligen Landesparlaments nach dessen Wahl - herausgenommen.

Unter dem Stichwort „falscher“ Argumente für die Direktwahl des Regierungschefs weist Decker mit Recht die Behauptung zurück, eine solche Verkürzung der Legitimationskette sei demokratischer als die parlamentarische Wahl - einerseits. Andererseits führt er als ein „richtiges“ Argument an, die Direktwahl harmoniere besser mit dem Ausbau und mit der aktuellen Popularität plebiszitärer Prozesse: Direktdemokratische Verfahren „von unten“ seien im präsidentiellen System „besser aufgehoben“ als im parlamentarischen, man müsse sich nicht dauernd um ihre Verträglichkeit mit dem letzteren streiten, eventuelle Blockaden zwischen einem volksgewählten Ministerpräsidenten und dem Parlament könnten durch plebiszitäre Verfahren aufgelöst werden; auch dränge sich die Direktwahl deshalb auf, weil es den Ländern nicht möglich sein werde, die plebiszitären Elemente „zurückzudrängen“5. Demnach soll die Direktwahl wohl doch näher zu dem stehen, was die Partizipationsdebatte dem Bürger verspricht - irgendwie „demokratischer“ als das missmutig hingenommene parlamentarische Verfahren.

In dem Plädoyer für den Systemwechsel zur Direktwahl sind Prämissen mitverarbeitet - teils ausdrücklich, teils auch inzident -, die einige Überlegungen politisch-praktischer Art nahelegen. Das betrifft die Bewertung und Gewichtung gewisser Elemente von Präsidialsystem und parlamentarischem Regierungssystem, die Eigenstaatlichkeit der Bundesländer, ihre und ihrer Parlamente Aufgabenstellung sowie die Bedeutung der Homogenität der Verfassungsordnungen in Bund und Ländern.

(2) Die präsidialen Regierungssysteme und ihre Varianten mit volksgewählten, an der Regierungsführung mehr oder weniger stark beteiligten Staatspräsidenten scheinen derzeit ihre problematischen Elemente stärker hervorzukehren. Ob USA oder Russland: Die Erwartungen an einen durch seine direkte Wahl kraftvollen, vom Parlament nicht abhängigen Präsidenten, jedoch in seinen Machtbefugnissen durch ein ebenfalls kraftvolles Gesetzgebungsorgan sowie durch ein Verfassungsgericht gezügelt und ausbalanciert, werden offenbar von anderen wirkmächtigen Tendenzen - mutmaßlich auch solchen der weltweiten Entwicklung des Mediensystems - überlagert und abgedrängt, und zwar in unterschiedliche Richtungen. Im US-Kongress hat das „party voting“ in einem Maße zugenommen, von dem sich die Bewunderer eines um wechselnde Mehrheiten der unabhängigen Volksvertreter verhandelnden Präsidenten nichts träumen ließen - und von dem die um Fraktionsdisziplin verhandelnden Geschäftsführer in deutschen Parlamenten vielleicht träumen könnten. Die Geschlossenheit der „caucuses“ von Republikanern und Demokraten führt mittlerweile zu haushalts-, wirtschafts- und gesellschaftspolitisch schädlichen Blockaden, die vom Präsidialsystem strukturell nicht, wohl aber vom parlamentarischen System mittels der Misstrauensdrohung eines um die nächsten Wahlen besorgten Parlaments gewöhnlich abgefangen werden können.

In Russland ist dergleichen bisher nicht eingetreten, weil sich der Präsident in der Regel auf eine erdrückende Majorität in Duma und Föderationsrat - und anscheinend zugleich auf eine willfährige Justiz - verlassen kann. Das bewirkt offenbar bei ihm die Vorstellung, er müsse sich vor allem mit einer außerparlamentarischen Opposition auseinandersetzen, wofür autoritäre Maßnahmen in Betracht kommen. Auch in diesem Fall gilt, dass die Konflikt-

4 Vgl. Frank Decker, a.a.O. (Fn. 2), S. 300 f.

5 Vgl. im Einzelnen ebenda, S. 301 ff., S. 304. 
lösung in einem parlamentarischen System mit der immerhin nicht ausschließbaren Abwahldrohung gegen den Regierungschef besser aufgehoben wäre. Auch der Blick etwa auf die Türkei oder - besonders aktuell und lehrreich - Tschechien zeigt bei allen unterschiedlichen Voraussetzungen ein ähnliches Phänomen: In der institutionellen Konkurrenz zwischen dem Parlament und einem von dessen Vertrauensvotum unabhängigen, seinerseits direkt gewählten präsidialen Regierungschef ist ein autoritärer Zug latent, der im Falle von ökonomischen, sozialen oder kulturellen Krisen manifest wird.

In Frankreich oder Polen wird unter wirtschafts- und sozialpolitischen Schwierigkeiten - in wiederum anderer Ausprägung, aber strukturell doch ähnlich - das Defizit einer aktiven Vermittlungsinstanz zwischen Volk und Staatsleitung deutlich, einer Instanz, die zur Vermittlung im Sinne der Krisenbewältigung auch tatsächlich entschlossen ist, weil der Regierungschef von der Parlamentsmehrheit als ihr Spitzenrepräsentant ins Amt gebracht wurde und deren politisches Schicksal daher von seinem Erfolg oder Misserfolg mit abhängt. Ein Blick zurück auf die Weimarer Republik mag hier erkenntnisfördernd sein: Wenn das Parlament einen Regierungschef - damals den Reichskanzler - und seine Amtsführung nicht institutionell von Anfang bis Ende mitverantworten muss, sondern sich lediglich durch Misstrauensvotum von ihm distanzieren kann, wird mit den gesellschaftlichen Konflikten weniger integrativ umgegangen.

Von den präsidentiellen und präsidentiell-parlamentarisch gemischten Systemen Lateinamerikas soll in diesem Zusammenhang nicht näher die Rede sein und schon gar nicht von den Versuchen in Nordafrika. In Umbruchsituationen rufen die Menschen auf den Straßen weniger nach einem Präsidenten als nach einem frei gewählten Parlament. Es gibt jedenfalls weder historisch noch aktuell besonders gute Gründe, das Präsidialsystem gegenüber dem parlamentarischen als bürgernäher, gewaltenteiliger, demokratischer oder sonstwie vorzugswürdig zu qualifizieren.

(3) Damit hängt das Verständnis der Funktionen zusammen, die den Parlamenten allgemein und den deutschen Landesparlamenten im Besonderen zugeschrieben werden. Historisch bildet die Gewaltenteilung zwischen „dem“ Parlament (als ganzem) und der Regierung die Hintergrundfolie dafür, dass als parlamentarische Hauptfunktion herkömmlich die Gesetzgebung betrachtet wird, und zwar umfassend in dem Sinne, dass die Initiative, das Programm, der Entwurf dazugehören. Das ist scheinbar fundiert durch den Sprachgebrauch des Grundgesetzes, wenn dort (Art. 20 Abs. 2 Satz 2) vom Bundestag als einem „Organ der Gesetzgebung" die Rede ist. An anderer Stelle (Art. 77 Abs. 1 Satz 1) ist das Grundgesetz deutlich näher an dem von ihm in den Artikeln 63, 67 und 68 eingerichteten parlamentarischen Regierungssystem: „Die Bundesgesetze werden vom Bundestag beschlossen. “6 Darauf kommt es an, nicht auf die Frage, wer sie planen, entwerfen, formulieren und dem Parlament vorlegen darf. Deshalb kommen die meisten Entwürfe von der durch die Parlamentsmehrheit installierten, legitimierten und mitkontrollierten Regierung, nur wenige „aus der Mitte des Bundestages" (Art. 76 Abs. 1 GG).

Keineswegs zufällig wird in den USA der Abgeordnete auch als „legislator“ bezeichnet. Die historisch auf der Monarchie aufsetzende Position des Präsidenten („Wahlkönig“) lässt mangels einer parlamentarischen Kontrolle ihm gegenüber kaum eine andere Konstruktion der Gewaltenteilung zu, als die Gesetzgebung (einschließlich der Gesetzgebung über den

6 Vgl. dazu auch Eberhard Schuett-Wetschky, Regierung, Parlament oder Parteien: Wer entscheidet, wer beschließt?, in: ZParl, 36. Jg. (2005), H. 3, S. 489 - 507. 
Staatshaushalt) zur Domäne der Volksvertretung zu erklären, ganz ähnlich wie in den konstitutionellen Monarchien Zentraleuropas im 19. Jahrhundert. Das parlamentarische Regierungssystem hat diese Form der "checks and balances“ hinter sich gelassen und reagiert damit - und kann damit überhaupt erst adäquat reagieren - auf die moderne pluralistische Gesellschaft, in der die von der Verfassung auszubalancierenden Machtpositionen heute gänzlich anders gelagert und gemischt sind als unter den Verhältnissen von Ständegesellschaft und Monarchie: Arbeitsteilung, Wertedifferenzierung, Wirtschaft, Massenmedien, Wissenschaft, Verbände usw.

Erst das parlamentarische Regierungssystem kann eine religiös oder ideologisch nicht mehr vorgeordnete, in Werten und Wünschen prinzipiell uneinige, in ihren Interessen kontroverse Gesellschaft so abbilden und in die Staatswillensbildung integrieren, dass sie mit sich selbst einig bleibt und dass allseits verbindliche Entscheidungen des Staates hinnehmbar sind. Hier liegt das Alleinstellungsmerkmal des Parlaments in diesem System. Es macht die Regierung (nicht statisch verstanden als Organ, sondern als politische Funktion) zum Bestandteil der Volksvertretung, indem es sie aus dieser hervorgehen und zu dieser zurückkehren lässt - jedes Mal, wenn zum Parlament und danach im Parlament gewählt wird.

(4) Von dieser Konstellation gibt es keinen Weg zurück zu Verhältnissen, in denen ein volksgewählter Ministerpräsident eine starke, vorzugsweise fachlich operierende Exekutive führt und der Landtag ihm gegenüber wie befreit aufspielt, indem er sich eigenhändig und initiativ um Entwurf und Einbringung der (in seiner Zuständigkeit verbliebenen) Gesetze kümmert. Gesetze zu ersinnen ist nicht die entscheidende Funktion der Landesparlamente - übrigens unter dem Aspekt der EU-Rechtsetzung auch zunehmend weniger die des Bundestages -, sondern die politische und öffentliche Kontrolle der Regierung, gerade auch hinsichtlich ihrer Regelungsvorhaben, nebst der Vermittlung dessen, was und warum da kontrolliert wird. Das geht über die schon 1867 für das britische Unterhaus von Walter Bagehot beschriebene ,informing function" ebenso weit hinaus wie über seine "teaching function ". 7 So wichtig die ist und so lange es gedauert hat, bis sie in Deutschland als bedeutende Parlamentsaufgabe begriffen wurde, so wenig würde die darin liegende Grundvorstellung - Parlament als Sender und politische Öffentlichkeit als Empfänger - den heutigen Erfordernissen genügen.

Es geht jetzt um mehr, und genau dabei können die Landesparlamente zu ihrer wirklichen und wirkungsvollen Rolle kommen: Unter den heutigen Bedingungen politischer Information, Interessenartikulation und Partizipation - einschließlich der davon logischerweise ausgelösten Komplexitätswahrnehmungen - hat das Parlament vor allem die Aufgabe, das Handeln des Staates diskutierbar, kritisierbar und erklärbar zu machen. Wenn es weiterhin „wählbar" bleiben will in einem fundamentalen Sinne als Vertretung des Souveräns, muss es die Verantwortung dafür auf sich nehmen, wie das Land regiert wird. Dafür ist das notwendig, was parlamentarische Kontrolle im parlamentarischen Regierungssystem hergibt, und zwar in beiden politischen Formen und Zielrichtungen: in der oppositionellen, kritischen, alternativen „contre-róle“ der Parlamentsminderheit und in der mitsteuernden und korrigierenden „control“ der regierungstragenden Parlamentsmehrheit. Dafür wiederum ist, als

7 Vgl. Walter Bagehot, The English Constitution, London 1867 (deutsch: Die englische Verfassung, herausgegeben und eingeleitet von Klaus Streifthau, Neuwied / Berlin 1971); zu Rang und Bedeutung der "Legitimation durch Kommunikation“ heute siehe vor allem Heinrich Oberreuter, Republikanische Demokratie. Der Verfassungsstaat im Wandel, Baden-Baden 2012, S. 137 und passim. 
Basis oder „fleet in being“ für alle kleinteiligeren Kontrollaktivitäten, die Wahl und Neuwahlmöglichkeit des Regierungschefs im Parlament notwendig.

Dabei spielt es keine Rolle, worin die Tätigkeiten der Landesregierungen im Einzelnen bestehen oder ob sie - wie Decker vorträgt ${ }^{8}$ - stärker mit kommunalen Aufgaben als mit solchen der Bundespolitik vergleichbar sind. Ob das zutrifft, wenn man die zunehmende Bedeutung europarechtlicher Regelungen für die Länder und die richtigerweise zunehmende Mitwirkung der Landesparlamente an den Aktivitäten ihrer Regierungen im Bundesrat berücksichtigt, mag hier dahinstehen. Auch soll offenbleiben, ob die Zuordnung zur Kommunalpolitik bei Ländern angezeigt ist, deren Einwohnerzahlen oft diejenigen zahlreicher EU-Mitgliedstaaten, teilweise um ein Mehrfaches, übertreffen. Die verfassungsrechtlich unaufhebbare Eigenstaatlichkeit der Bundesländer (Art. 79 Abs. 3 GG) spricht sicher nicht dafür, ihre Regierungen assoziativ in die Nähe einer Provinzialverwaltung zu rücken. Dafür gibt auch die beliebte Redensart nichts her, es gebe „keinen christdemokratischen Straßenbau und keine sozialdemokratische Wasserwirtschaft" (mit der laut Decker die „Eigenart der Landespolitik“ durch Hennis „,auf den Punkt gebracht“ worden sei ${ }^{9}$ ). Selbstverständlich gibt es die, und genau das ist es, was die Parteien, und zwar ganz besonders in den Parlamentsfraktionen, herausarbeiten und vermitteln müssen: was es politisch bedeutet und bewirkt, wenn mehr oder weniger Finanzmittel für den Straßenbau ausgegeben und wenn Wasserwerke privatisiert oder in öffentlicher Hand betrieben werden. Den Bürgern vorzumachen, es gehe da nur um Sachfragen und Sachzwänge, und statt der vermaledeiten „Parteipolitik“ müsse den „Experten“ gefolgt werden, gehört zu den Attitüden, mit denen politisches Verständnis und politische Bildung untergraben werden. Interessenprüderie und Parteiaversion sind nicht imstande, in einer freien, pluralistischen, in Interessen und Erwartungen kontroversen Gesellschaft substanzielle politische Mitwirkung zu fördern.

(5) Für die Stellung der Länder im föderalen System bleibt entscheidend, dass sie als Gliedstaaten nicht nur verfassungsrechtlich figurieren, sondern auch politisch auftreten. Das ist angesichts ihrer gewachsenen Einbindung in bundespolitisch, europapolitisch und auch weltpolitisch wirksame Entwicklungen heute nicht weniger, sondern mehr notwendig. Je weniger übersichtlich die politischen Handlungsbedingungen erscheinen, desto mehr ist der öffentliche Diskurs gefordert. Nur dadurch kann der Verkehr mit den Bürgern darüber aufrecht erhalten werden, was der Regierungschef in den verschiedenen politischen Arenen für sie tut oder tun sollte. Die hier mehr denn je nötige Dauerkommunikation sollte besser nicht Regierungssprechern und Presseabteilungen überlassen werden. Eine verbindliche und systematische Kontrolle des Regierungshandelns ist von den Parlamenten zu leisten, und zwar diskursiv und mit feedback-Schleifen. Dafür müssen sie institutionellen Einfluss auf das Amt „ihrer“ Ministerpräsidenten haben - und dadurch dann auch auf das, was diese mit anderen Landesregierungen, mit dem Bund und in Brüssel machen.

Es geht mithin um einen kooperativen Beitrag der Länder zur Integration zwischen gesellschaftlicher und staatlicher Willensbildung im föderalen Gesamtstaat. Genau darin liegt

8 Vgl. Frank Decker, a.a.O. (Fn. 2), S. 302.

9 Vgl. ebenda, S. 301 unter Hinweis auf die in der dortigen Fußnote 3 zitierte Abhandlung von Wilhelm Hennis, Parlamentarische Opposition und Industriegesellschaft. Zur Lage des parlamentarischen Regierungssystems, in: Gesellschaft - Staat - Erziehung, 5. Jg. (1956), H. 1, S. 205 222, wiederabgedruckt in: ders., Regieren im modernen Staat. Politikwissenschaftliche Abhandlungen I, Tübingen 1999, S. 1 - 23. 
die tiefere Rechtfertigung für ihre Eigenstaatlichkeit: Sie bildet ein vertikales Element demokratischer Gewaltenteilung.

Das führt zu der Frage nach übereinstimmenden Regierungssystemen in Bund und Ländern. Die Weimarer Verfassung hatte in ihrem Artikel 17 für die „innere Ordnung der Länder“ statuiert: „Die Landesregierung bedarf des Vertrauens der Volksvertretung.“ Das Grundgesetz enthält in Art. 28 über die „verfassungsmäßige Ordnung der Länder“ eine solche Bestimmung nicht, sondern nur die Vorgabe, sie müsse „den Grundsätzen des republikanischen, demokratischen und sozialen Rechtsstaats im Sinne dieses Grundgesetzes entsprechen“. Ob dieses so genannte Homogenitätsgebot das parlamentarische Regierungssystem nach GG-Muster umfasst, ein Präsidialsystem in den Ländern demzufolge als verfassungswidrig qualifiziert werden könnte, muss einer vertieften Diskussion überlassen bleiben, die hier nicht geführt werden kann. Das Bundesverfassungsgericht hatte schon am Beginn seiner Rechtsprechung die parlamentarische Verantwortlichkeit der Regierung einerseits zu den Essenzialien der freiheitlich demokratischen Grundordnung gezählt und dies später bekräftigt, andererseits aber auch mehrfach betont, das Homogenitätsgebot fordere keine Konformität oder Uniformität. In der Literatur wird sowohl der Grundsatz der Rechtseinheit des Gesamtstaats als auch das selbständige Nebeneinander von Bundes- und Landesverfassungen hervorgehoben. Die rechtliche Frage nach der Reichweite von Art. 28 Abs. 1 Satz 1 GG (die bei Decker, wohl im Hinblick auf ihre wissenschaftliche Unabgeschlossenheit, nicht behandelt wird) ließe sich letztlich nur in einem konkreten Fall klären, nämlich in einem Verfassungsstreit zwischen dem Bund und einem Land - oder auch zwischen einem Land und (einem) anderen - um die Einführung der Direktwahl.

In diesem Punkt erlaubt aber die diskursive Modernität des parlamentarischen Regierungssystems doch einen Schluss: Für übereinstimmende parlamentarische Regierungssysteme in Bund und Ländern liegen bessere Gründe vor als für divergierende. Es ist nicht ersichtlich, welchen demokratischen Fortschritt für Deutschland ein Landesparlament bringen sollte, das im Gegensatz zu anderen nicht mehr zur Regierungsbildung befugt wäre den Bürgern dieses Landes müsste zusätzlich erklärt werden, warum sie sich an seiner Wahl beteiligen sollten. 